\title{
Physician perspectives about the US Quality Payment Program: MIPS benefits and alternative payment model incentives
}

\author{
Joshua M. Liao ${ }^{1,2,3}$, Amol S. Navathe $\mathrm{e}^{3,4,5}$ \\ ${ }^{1}$ Department of Medicine, University of Washington School of Medicine, Seattle, WA, USA; ${ }^{2}$ Value \& Systems Science Lab, Seattle, WA, USA; \\ ${ }^{3}$ Leonard Davis Institute of Health Economics, Philadelphia, PA, USA; ${ }^{4}$ Department of Medical Ethics and Health Policy, Perelman School of \\ Medicine at the University of Philadelphia, PA, USA; ${ }^{5} \mathrm{CMC}$ Philadelphia VA Medical Center, Philadelphia, PA, USA \\ Correspondence to: Joshua M. Liao, MD, MSc, FACP. Department of Medicine University of Washington School of Medicine, 1959 Pacific Street NE, \\ BB1240, Box 356526, Seattle, WA 98195, USA. Email: joshliao@uw.edu.
}

Background: In the US, Medicare has implemented a nationwide Quality Payment Program via two
participation options-the Merit-based Incentive Payment System (MIPS) and advanced alternative payment
models (APMs) - to reform physician reimbursement. Program success depends on physician engagement,
which in turn depends on how physicians believe the MIPS will impact care and whether they are willing to
accept new APM incentives. Methods: We conducted a national web-based survey of 1,431 practicing physicians to evaluate their perspectives about (I) how the MIPS would ultimately impact stakeholder groups and (II) willingness to accept incentives related to major advanced APMs [accountable care organizations (ACOs), bundled payments, risk-adjusted capitation].

Results: Of 1,431 eligible physicians, 51\% responded. Few believed that the MIPS would ultimately benefit any stakeholder groups. For instance, the minority reported the belief that the MIPS would benefit Medicare patients (17\%) or physicians (8\%) and hospitals/clinics (13\%) caring for them, and more physicians believed the MIPS would ultimately harm these groups. With regards to APM incentives, less than a third of physicians reported willingness to accept risk-adjusted capitation (28\%), shared savings/loss (23\%), or bundled payment $(23 \%)$ arrangements even in exchange for a guaranteed $5 \%$ increase in reimbursement from their largest payer.

Conclusions: Our findings underscore concerns expressed about the MIPS and spotlight additional work needed to increase physician willingness to engage in advanced APMs.

Keywords: Health policy; value-based payment; Quality Payment Program (QPP); alternative payment models (APMs); Merit-based Incentive Payment System (MIPS)

Received: 02 December 2019. Accepted: 05 March 2020; Published: 25 June 2020.

doi: $10.21037 /$ jhmhp.2020.03.04

View this article at: http://dx.doi.org/10.21037/jhmhp.2020.03.04

\section{Introduction}

In 2017, Medicare implemented the Quality Payment Program (QPP) as a key strategy for shifting the US health care system away from fee-for-service reimbursement toward value-based payment (1). Most physicians who currently care for Medicare beneficiaries nationwide are required to participate in the QPP via one of two tracks.

In the first track, the Merit-based Incentive Payment
System (MIPS), physicians are evaluated annually based on activities in the four domains of Quality, Improvement Activities, Cost, and Promoting Interoperability. Performance in each domain is weighted and the resulting scores are combined across domains to determine upward or downward adjustments to physicians' overall professional payments. These adjustments can be significant, ranging from $\pm 5 \%$ in 2020 to $\pm 9 \%$ in 2022 and beyond.

The alternative to MIPS is the Alternative Payment 
Model (APM) track, in which physicians participate in the QPP by enrolling in at least one value-based APM that is "advanced" by virtue of involving the potential for financial loss (e.g., payment decreases or shared losses), not just financial gain (e.g., bonus payments, shared savings). Physicians can qualify for the APM track by electing to participate in a number of eligible arrangements, with the most prominent nationwide examples including accountable care organizations (ACOs) as a population-based payment model, bundled payment as an episode-based payment model, and emerging risk-adjusted capitation arrangements such as direct contracting models ) (2-4).

The success of the QPP may rest on how physicians view and engage with its two participation tracks. In particular, physicians' willingness to engage in MIPS likely depends in part on which stakeholders - patients, providers, or othersthey believe benefit from the program. Moreover, physician willingness to accept advanced APM incentives over fee-forservice incentives is likely a key determinant of engagement and participation in advanced APMs. Because MIPS is intended to serve as a transitional path toward advanced APMs, it is critical to understand perspectives about both the benefits of MIPS as well as advanced APM incentives.

Despite the importance of these issues-if and how physicians believe MIPS benefits different stakeholders; whether physicians would accept APM incentives-little is known about them.

\section{Methods}

Between March and May of 2017, we conducted a webbased survey using a national sample of 1,431 practicing internal medicine physicians maintained by the American College of Physicians (ACP) (5), the methods and design of which have been previously described in detail elsewhere (6). As part of that survey, respondents were asked how MIPS would ultimately impact (Benefit, Harm, Neither, Not Sure) the following stakeholder groups: Medicare patients, physicians caring for Medicare patients, hospital/clinics accepting Medicare patients, Medicare itself, and society.

In a separate question, respondents were also asked whether they "would accept each of the following payment arrangements in exchange for a guaranteed $5 \%$ increase in reimbursement from their largest payer": (I) Shared Savings/Loss (with opportunity to gain or lose up to $2 \%$ of fee-for-service payments based on performance against a yearly spending benchmark); (II) Bundled Payment (with opportunity to gain or lose up to $2 \%$ of fee-for-service payments based on performance against an episode spending benchmark); (III) Risk-adjusted Capitation/Per-Member Per-Month payments (with performance incentive based on quality and utilization metrics). By using example payment arrangements that correspond to Medicare Advanced APMs (e.g., ACOs as a shared savings/loss arrangement; Directing Contracting as a capitation arrangement), these questions were designed to evaluate physicians' willingness to accept APM incentives. Survey responses were described using percentages. Analyses were performed using STATA version 14.1 (STATA Corp, College Station, TX, USA). The protocol was reviewed and deemed to be exempt by the University of Pennsylvania Institutional Review Board.

\section{Results}

Of 1,431 eligible participants, 51\% $(\mathrm{n}=726)$ returned complete or partial surveys. As noted previously, over half (51\%) were men and general internal medicine physicians (primary care, hospital medicine, geriatrics; $71 \%$ ), with a median age of 48 (IQR 39-58). Compared to nonrespondents, respondents were more likely to be male $(51 \%$ versus $42 \%)$ and older (mean age 49 versus 43$)(\mathrm{P}<0.001$ for both).

\section{Impact of MIPS on stakebolders}

Few physicians believed that MIPS would ultimately benefit any stakeholder groups (Figure 1). In particular, the minority reported the belief that MIPS would benefit Medicare patients $(17 \%)$, society $(13 \%)$, physicians $(8 \%)$ and hospitals/clinics (13\%) caring for Medicare patients, and even Medicare itself (27\%). In contrast, larger proportions of physicians reported the belief that MIPS would ultimately harm these groups. For instance, approximately half believed the program would harm physicians (55\%) and hospitals/clinics (49\%) caring for Medicare patients, while a quarter to a third of respondents believed MIPS would harm Medicare patients (33\%), society (32\%), and the Medicare program itself (24\%). The only group that respondents believed would experience more benefit than harm was Medicare itself ( $27 \%$ believing Medicare would benefit from MIPS versus $24 \%$ believing it would be harmed by MIPS).

\section{Willingness to accept advanced APM incentives}

Overall, the minority of respondents reported willingness to 


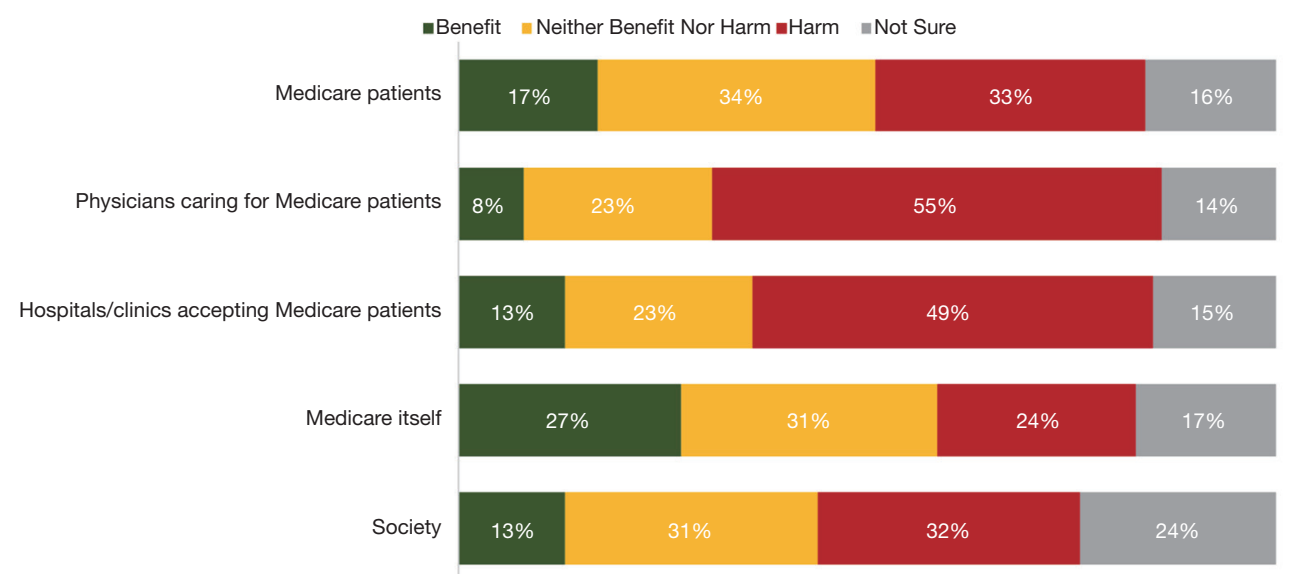

Figure 1 Physician perspectives about the impact of MIPS on stakeholders Groups. This figure shows responses from 726 physicians to the following question: "In your opinion, will such a Medicare merit-based payment system ultimately benefit or harm each of the following?". Answer options included Benefit, Harm, Neither Benefit Nor Harm, and Not sure. MIPS, Merit-based Incentive Payment System.

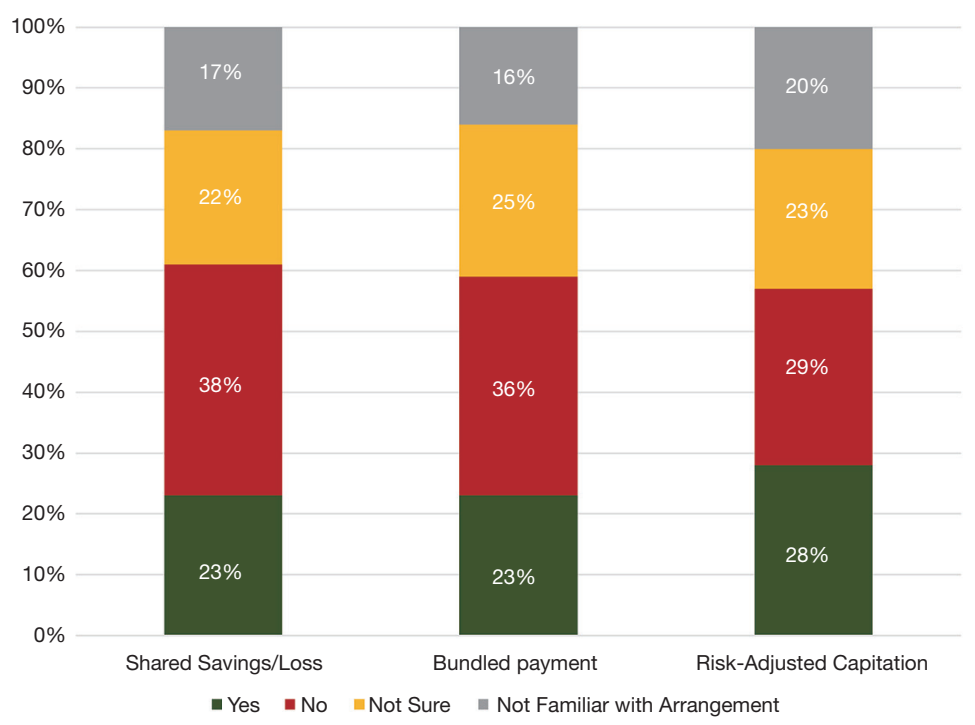

Figure 2 Willingness to accept APM incentives. This figure shows responses from 726 physicians to the following question: "If your largest payer guaranteed a $5 \%$ increase in reimbursement in return, would you accept each of the following payment arrangements?". Answer options included Yes, Not, Not Sure, and Not Familiar with Arrangement. APM, alternative payment model.

accept incentives related to major advanced APMs (Figure 2). In particular, less than a third reported willingness to accept risk-adjusted capitation (28\%), shared savings/loss (23\%), or bundled payment (23\%) arrangements even in exchange for a guaranteed $5 \%$ increase in reimbursement from their largest payer. Approximately a third of respondents reported not being willing to accept these incentives: $38 \%$ for shared savings/loss, $36 \%$ for bundled payment, and $29 \%$ for risk- adjusted capitation arrangements. A quarter of respondents were unsure (22-25\%), and fewer were unfamiliar with these arrangements (16-20\%).

\section{Conclusions}

In a survey evaluating physician perspectives about a large national value-based payment program in the US, we 
provide insight into how practicing physicians believe MIPS will impact various stakeholder groups, as well as physician willingness to accept advanced APM incentives. Our findings pose several policy implications.

First, our results underscore the concerns that physicians and other health care stakeholders possess about MIPS. Though existing evidence suggests that practicing physicians and medical trainees tend to have positive perspectives about the program in general $(6,7)$, a notable proportion also possess concerns about the ability for MIPS to improve value and the risk of creating unintended consequences. Other stakeholders such as MedPAC have criticized the program, advocating Medicare to end the program and use a voluntary alternative its place (8). The fact that few physicians in our sample believed MIPS would benefit stakeholder groups reinforces the opportunity for policymakers to continue improving MIPS through stakeholder engagement and dialogue-an explicit focus adopted by Medicare in MIPS policy (1).

Second, our results spotlight additional work needed in order to increase physician willingness to engage in advanced APMs. Despite increased APM participation being a key goal of QPP, only a minority of physicians in our study reported being willing to accept incentives related to ACOs, bundled payments and risk-adjusted capitation, in exchange for a guaranteed $5 \%$ payment increase. This hesitation may relate in part to a lack of familiarity with these payment models-16-20\% of respondents reported being unfamiliar with the arrangements-and in part to concerns about the payment arrangements themselves (e.g., uncertainty about the ability to perform well in APMs; concerns about compliance and reporting burden associated with participation). Regardless, future work should explore provider perspectives about APMs in order to understand factors driving willingness to participation.

Our study has limitations. First, the generalizability of our findings is limited by response rate, though our findings remain relevant as what is to our knowledge the first description of physician perspectives of these two key aspects of the QPP. Second, while we did not evaluate physician perspectives on a comprehensive list of advanced APMs, we focused on what the three most prominent arrangements nationwide as defined by current and emerging Medicare policy. Third, we assessed overall perspectives about how MIPS would benefit or harm stakeholder groups, but not specific drivers of benefit or harm. This remains an important area for future work. Fourth, though our study evaluated early physician perspectives in the first year of the QPP, the program has been stable over several years with few changes to MIPS and major advanced APMs.

Nonetheless, our study supports ongoing payment policy by shedding light on how physicians view MIPS and advanced APM incentives-key factors that will dictate the success of the nationwide QPP. Our findings offer insight to policymakers seeking to using the QPP to drive greater value in the US health care system.

\section{Acknowledgments}

The authors would like to acknowledge Arlene Weissman, $\mathrm{PhD}$, for assistance with data collection.

Funding: None.

\section{Footnote}

Conflicts of Interest: Both authors have completed the ICMJE uniform disclosure form (available at http://dx.doi. org/10.21037/jhmhp.2020.03.04). JML reports other from Wolters Kluwer, personal fees from Kaiser Permanente Washington Research Institute, outside the submitted work; ASN reports grants from Hawaii Medical Service Association, grants from Anthem Public Policy Institute, grants from Commonwealth Fund, grants from Oscar Health, grants from Cigna Corporation, grants from Robert Wood Johnson Foundation, grants from Donaghue Foundation, grants from Pennsylvania Department of Health*, personal fees from Navvis Healthcare, personal fees from Agathos, Inc., personal fees from Navahealth, personal fees from Embedded Healthcare, personal fees from University Health System - Singapore, personal fees from Social Security Administration - France, personal fees from Medicare Payment Advisory Commission, personal fees from Elsevier Press, personal fees from Cleveland Clinic, other from Integrated Services, Inc., outside the submitted work.

Ethical Statement: The authors are accountable for all aspects of the work in ensuring that questions related to the accuracy or integrity of any part of the work are appropriately investigated and resolved. The protocol (\#826825) was reviewed and deemed to be exempt by the University of Pennsylvania Institutional Review Board.

Open Access Statement: This is an Open Access article distributed in accordance with the Creative Commons 
Attribution-NonCommercial-NoDerivs 4.0 International License (CC BY-NC-ND 4.0), which permits the noncommercial replication and distribution of the article with the strict proviso that no changes or edits are made and the original work is properly cited (including links to both the formal publication through the relevant DOI and the license). See: https://creativecommons.org/licenses/by-nc-nd/4.0/.

\section{References}

1. MACRA. Centers for Medicare \& Medicaid Services. Accessed 1 December 2019. Available online: https://www. cms.gov/Medicare/Quality-Initiatives-Patient-AssessmentInstruments/Value-Based-Programs/MACRA-MIPS-andAPMs/MACRA-MIPS-and-APMs.html

2. Next Generation ACO Model. Centers for Medicare \& Medicaid Services. Accessed 1 December 2019. Available online: https://innovation.cms.gov/initiatives/nextgeneration-aco-model/

3. BPCI Advanced. Centers for Medicare \& Medicaid

doi: 10.21037/jhmhp.2020.03.04

Cite this article as: Liao JM, Navathe AS. Physician perspectives about the US Quality Payment Program: MIPS benefits and alternative payment model incentives. J Hosp Manag Health Policy 2020;4:16.
Services. Accessed 1 December 2019. Available online: https://innovation.cms.gov/initiatives/bpci-advanced

4. Direct Contracting Model Options. Centers for Medicare \& Medicaid Services. Accessed 1 December 2019.

Available online: https://innovation.cms.gov/initiatives/ direct-contracting-model-options/

5. American College of Physicians. Accessed 1 December 2019. Available online: https://www.acponline.org/

6. Liao JM, Shea JA, Weissman A, et al. Physician Perspectives In Year 1 Of MACRA And Its MeritBased Payment System: A National Survey. Health Aff (Millwood) 2018;37:1079-86.

7. Liao JM, Shea JA, Weissman A, et al. US trainee perspectives on a national pay-for-performance program. J Hosp Manag Health Policy 2019;3:26.

8. Dickson V. MedPAC vots 14-2 to junk MIPS, providers angered. Modern Healthcare. Accessed 1 December 2019. Available online: https://www.modernhealthcare.com/ article/20180111/NEWS/180119963/medpac-votes-14-2to-junk-mips-providers-angered 